\title{
Changing Scenario in Drug Delivery Systems and Future Prospects
}

\section{Rajesh Singla*}

Associate Professor, Microbiology and Head, Agriculture Department, S.S.D. College of Professional Studies, Punjab, India

*Corresponding Author: Rajesh Singla, Associate Professor, Microbiology and Head, Agriculture Department, S.S.D. College of Professional Studies, Punjab, India.

Received: July 11, 2019; Published: July 31, 2019

DOI: $10.31080 /$ ASPS.2019.03.0361

Novel Drug delivery System (NDDS) involves various technological approaches and systems for delivering a drug (pharmaceutical compound) or a formulation in a minimum required dose to the desired site of action safely and effectively so as to get maximum results. NDDS covers various aspects like pharmacokinetics and pharmacodynamics, non-specific toxicity, immunogenicity, bio-recognition, drug's efficacy based on approaches that includes polymer science, pharmaceutical technology, dispensing pharmacy, packaging technology, bioconjugate chemistry and molecular biology.

In the novel drug delivery systems (NDDS), there are various novel carriers which have advantage over conventional dosage forms. The method of drug delivery has a significant effect on its efficacy (dose, degradation, loss, bio-availability, side effects etc.). The choice of a drug delivery route is driven by the properties of the drug, acceptance of the drug by the patient for example its solubility, access to the location where disease has occurred and effectiveness of the drug in dealing with the specific disease. There are two kinds- active targeted drug delivery such as antibody medications and passive targeted drug delivery such as the enhanced permeability and retention effect. Extensive research is going on to incorporate herbal drugs in the novel drug delivery systems. Application of these novel techniques to natural medicines will lead to enhanced bioavailability, reduced toxicity, sustained release action, protection from gastro-intestinal degradation which cannot be obtained through conventional drug delivery system due to large molecular size, poor solubility and degradation of herbal medicines in gastrointestinal media.

NDDS is one of the important tool expanding drug markets in pharmaceutical industry. The global market for advanced drug delivery systems amounted to $\$ 134.3$ billion in 2008 . The estimate for 2014 is $\$ 196.4$ billion, for a compound annual growth rate (CAGR) of $7.2 \%$ in the 5 -year period. The novel drug delivery systems market is expected to witness a CAGR of $2.9 \%$, during the period 2018-2023. There is a continuous growth in the market of drug delivery systems and is forecasted to continue to grow at an impressive rate in future also.

The stability of the delivery system is one of the most important factors, which affects the drug delivery systems market. Nanotechnology became the driving force behind various evolutionary changes in the scientific field. Nanotechnology based drug delivery enhanced the efficacy of available medicaments and enabled to create entirely new medical products. Nanotechnology has opened the door to new inventions to stimulate the reconstruction of complex tissue structures. The global nanotechnology drug delivery market was valued at US\$ 41,062.5 Mn in 2014 and is projected to reach US\$118,527.2 Mn by 2023, expanding at a CAGR of $12.5 \%$ from 2015 to 2023. Nanoparticles and nanoformulations are of current interest because of an emerging understanding of their possible effects on human health and environmental sustainability, and owing to the increasing output of man-made nanoparticles into the environment which includes including anti-tumor therapy, gene therapy, AIDS therapy, radiotherapy, in the delivery of proteins, antibiotics, virostatics, vaccines and as vesicles to pass the blood - brain barrier.

Owing to the several advantages over other delivery systems, Liposomes are the most common nano-carriers used for drug delivery. Liposomes not only help in stabilizing therapeutic compounds and but also improves in vivo distribution of compounds to the target sites. However, one of the major concerns restraining the growth of this market is stability of liposomebased drug delivery systems along with some other factors which includes stringent regulatory guidelines and product recalls.

\section{Volume 3 Issue 8 August 2019 \\ (C) All rights are reserved by Rajesh Singla.}

